\title{
Risk factors for pre-eclampsia and eclampsia in Guatemala
}

Population Council

Follow this and additional works at: https://knowledgecommons.popcouncil.org/departments_sbsr-rh

Part of the Demography, Population, and Ecology Commons, Family, Life Course, and Society Commons, International Public Health Commons, Maternal and Child Health Commons, and the Women's Health Commons How does access to this work benefit you? Let us know!

\section{Recommended Citation}

"Risk factors for pre-eclampsia and eclampsia in Guatemala," fact sheet. Washington, DC: Population Council, 2017. 
Each day around the world, 830 women die from pregnancy- and childbirth-related causes. The second most common cause (after postpartum hemorrhage) is a hypertensive disorder during pregnancy, such as pre-eclampsia and eclampsia (PE/E)-life-threatening, pregnancyinduced high blood pressure and excess protein in urine-which can lead to seizures and other fatal complications. One in four preterm infants dies as a result of their mother's PE/E. These deaths are preventable, yet essential medicines and tools to treat this disorder are often unavailable in low-resource settings.

\section{DEMOGRAPHICS}

POPULATION:
16.6 MILLION
TOTAL FERTILITY RATE:
3.1 BIRTHS
PER WOMAN
UNMET NEED FOR
POSTPARTUM
CONTRACEPTION:
13.9\%

\section{OVERARCHING HEALTH RISKS}

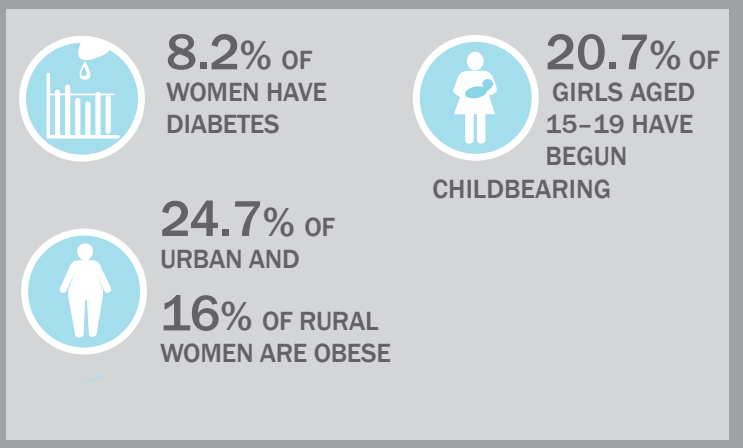

\section{BARRIERS TO ACCESSING SERVICES}

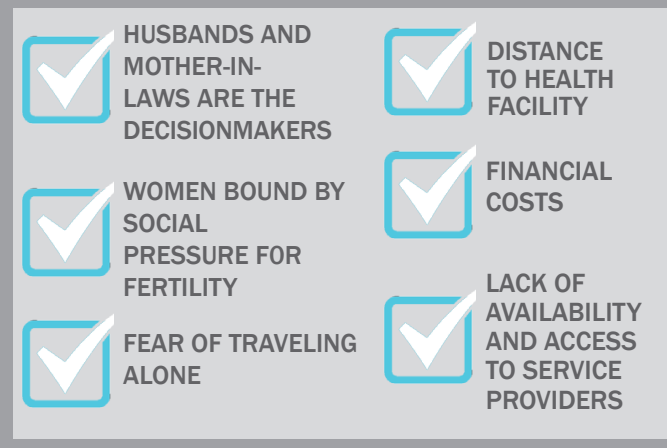

\section{PREGNANCY-RELATED CARE}

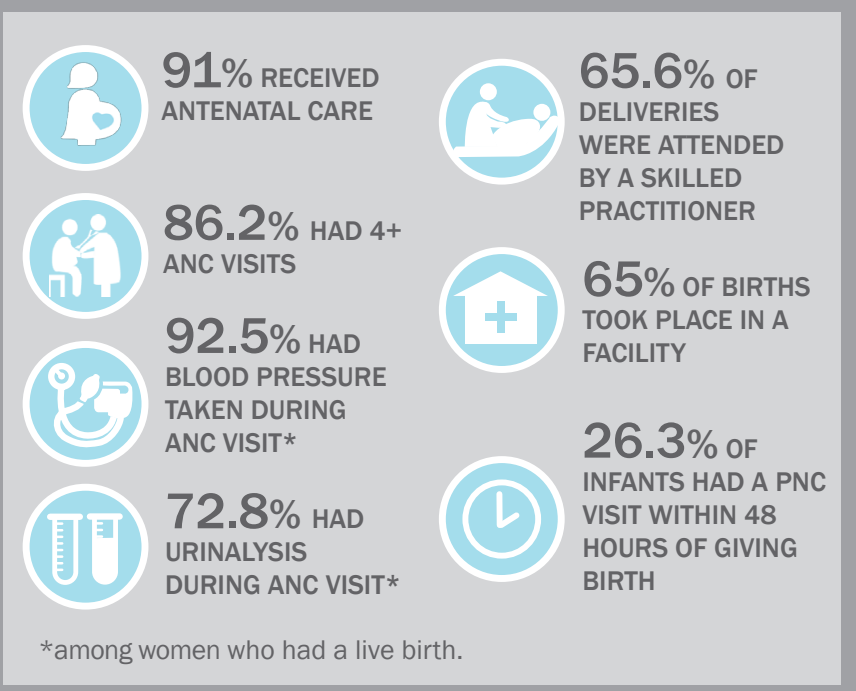

MATERNAL DEATH DATA

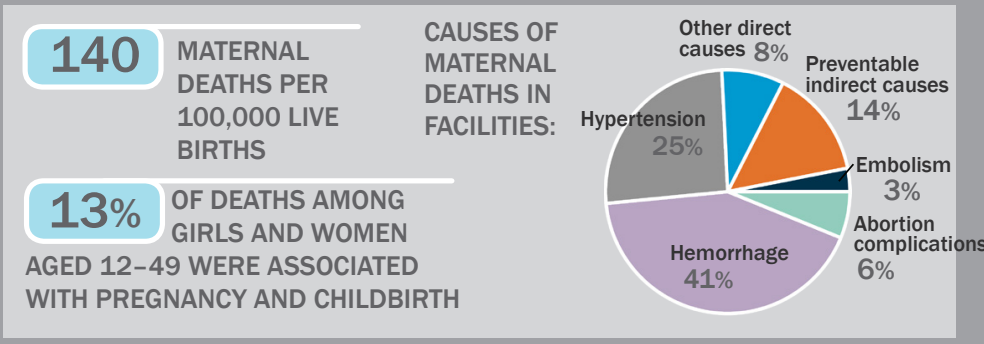

\section{NEWBORN/INFANT DEATH DATA}

\begin{tabular}{|l|l}
28 & $\begin{array}{l}\text { INFANT DEATHS } \\
\text { PER 1,000 LIVE } \\
\text { BIRTHS }\end{array}$ \\
17 & $\begin{array}{l}\text { NEONATAL DEATHS } \\
\text { PER 1,000 LIVE } \\
\text { BIRTHS }\end{array}$ \\
\hline
\end{tabular}

\begin{tabular}{ll}
22 & $\begin{array}{l}\text { PERINATAL DEATHS } \\
\text { PER 1,000 } \\
\text { PREGNANCIES }\end{array}$ \\
$12 \%$ & $\begin{array}{l}\text { OF NEONATAL DEATHS } \\
\text { WERE RELATED TO } \\
\text { PRETERM BIRTH }\end{array}$ \\
\hline
\end{tabular}

\section{$\mathrm{MgSO}_{4} / \mathrm{CG}$ DELIVERY CAPACITY}

\section{SPECIALISTS, MEDICAL OFFICERS, LADY HEALTH} VISITORS, MEDICAL TECHNICIANS, AND DISPENSERS CAN ADMINISTER $\mathrm{MgSO}_{4}$ AND CALCIUM GLUCONATE (CG)

\section{NO DATA OF NON-TEACHING HOSPITALS HAVE $\mathrm{MgSO}_{4}$ AND CG IN STOCK}

(ALL TEACHING HOSPITALS HAVE BOTH IN STOCK)

\section{NO DATA OF STAFF ARE TRAINED TO}

ADMINISTER $\mathrm{MgSO}_{4}$ AND CG

\section{NATIONAL/STATE POLICIES}

Of the 13 UN Lifesaving Commodities for Women and Children, which are on the national essential medicines list?

Which antihypertensives are on the national essential medicines list?

Are there formal mechanisms for procuring these drugs?

Is there a task-shifting policy in country?
Oxytocin, misoprostol, $\mathrm{MgSO}_{4}$, injectable antibiotics, chlorhexidine, amoxicillin, calcium gluconate, oral rehydration salts, zinc, contraceptive implants

Hydralazine and methyldopa Sources: Ministerio de Salud Pública y Asistencia Social (MSPAS), Instituto Nacional de Estadística (INE), ICF International, 2017, Encuesta Nacional de Salud Materno Infantil 2014-2015, Informe Final. Guatemala MSPAS/INE/ICF. Encuesta Nacional de Condiciones de Vida, 2014, Guatemala 2016. The World Bank Data, Adolescent fertility rate, 2017. UN Commission on Life-Saving Commodities for Women and Children, Commissioners' Report, September 2012 Lista Basica de Medicamentos Ministerio de Salud Publica y Asistencia Social, Guatemala, 2013. Informatica y Vigilancia Epidemiologica, Guatemala, 2004. Memoria de labores, Ministerio de Salud Publica y Asistencia Social, 2016. WHO Diabetes Country Profile, Guatemala, 2016. Estadística de Mortalidad materna, Guatemala, enero a diciembre 20142015, 2016. 